\title{
A Repeated Convex Fuzzy Cooperative Game
}

\author{
Zuofeng Gao \\ Department of Science, Yanshan university \\ Qin Huangdao 066004, China \\ Yongbo Yu (Corresponding author) \\ Department of Science, Yanshan University \\ Qinhuangdao 066004, China \\ E-mail: yongbo12345@163.com \\ Hongxin Bai \\ Department of Science, Yanshan University \\ Qinhuangdao 066004, China \\ Chunyan Han \\ Department of Science, Yanshan university \\ Qin Huangdao 066004, China
}

Supported by the Foundation for the natural science of Hebei province of China (A2005000301)

\begin{abstract}
This paper introduces repeated theory on the base of fuzzy cooperative game by Aubin etal in 1974 and then constructs repeated fuzzy games theory. It gives the conception of repeated convex fuzzy cooperative games and studies the property of repeated convex fuzzy games.
\end{abstract}

Keywords: Fuzzy game, Convex, Repeated cooperative game

\section{Introduction}

The theory of fuzzy cooperative game had been studying widely and deeply since it was introduced by Aubin(1974, 1981), and Jorge ovido (1999) studied the theory of a repeated cooperative games. In this paper, we combine the two theory and construct a repeated fuzzy cooperative game.

The purpose of this paper is on one hand to present a detailed characterization of repeated convex fuzzy games and on the other hand to study the property of repeated convex fuzzy games.

\section{Fuzzy cooperative games and convex fuzzy cooperative games}

Let $N=\{1,2 \cdots \cdots n\}$ be a nonempty set of players considering possibilities of cooperation. A fuzzy coalition is a vector $S \in[0,1]^{N}$. The ith coordinate $S_{i}$ of $\mathrm{S}$ is called the participation level of player $i$ in fuzzy coalitions $\mathrm{S}$. For each crisp coalition $\mathrm{S}$, its characteristic vector is $e^{S}$, with $\left(e^{S}\right)_{i}=1$ if $i \in S ;\left(e^{S}\right)_{i}=0$, if $i \in N / S$.

A fuzzy game with player set $N$ is a map $v: F^{N} \rightarrow R$ with the property $v(0)=0$, The map $\mathrm{V}$ assigns to each fuzzy coalition can achieve in cooperation. The set of fuzzy games with player set $\mathrm{N}$ will be denoted by $F G^{N}$.

Definition 1: Let $v \in F G^{N}$. Then $v$ is called a convex fuzzy game if function $v:[0,1]^{N} \rightarrow R$ is a supermodular and coordinate-wise convex function on $[0,1]^{N}$. (see reference R.Branzei (2003)) 


\section{Repeated fuzzy cooperative games}

We repeat $\mathrm{m}$ times ( $\mathrm{m}$ may be $\infty$ ) this fuzzy cooperative games, we denote by $\left([0,1]^{N}, v_{t}, t\right)$ the stage fuzzy cooperative games $(N, v)$ that agents play in period t. The characteristic function of the game $\left([0,1]^{N}, v_{t}, t\right)$ is defined for all $S \in[0,1]^{N}$ by $V_{t}(S)=(1-\delta) \delta^{t} V(S)$.

We define the repeated fuzzy cooperative game, so that it has the same structure as the fuzzy cooperative game.

Definition 2 Let $S^{t} \subseteq[0,1]^{N}$ denote a coalition formed at time t .Let $\theta=\left(S^{0}, S^{1}, \cdots S^{t}\right)$

$=\left(S^{t}\right)_{0}^{m} \in H$ be a fuzzy coalition sequence, we define the repeated fuzzy characteristic function $\mathrm{w}$ as $W(\theta)=(1-\delta) \sum_{t=0}^{m} \delta^{t} V\left(S^{t}\right)$

we denote by $\mathrm{H}$ the set of all coalition sequences.

Definition3: A tuple $(\mathrm{H}, \mathrm{w})$ is repeated fuzzy cooperative games, where $\mathrm{H}$ is a fuzzy coalition sequence, $\mathrm{w}$ is the repeated fuzzy characteristic function defined by (1) The set of repeated fuzzy games with player set $\mathrm{N}$ will be denoted by $\left(F G_{t}^{N}\right)_{t=0}^{m}$.

\section{Main result}

Let $\theta=\left(S^{t}\right)_{t=0}^{m} \in H, \quad \psi=\left(T^{t}\right)_{t=0}^{m} \in H$ be two coalition sequences such that for all $0 \leq t \leq m, S^{t} \cap T^{t}=\phi, \theta \cup \psi=\left(S^{t} \cup T^{t}\right)_{t=0}^{m}$, $\theta \cap \psi=\left(S^{t} \cap T^{t}\right)_{t=0}^{m}$, then

Theorem 1: If $v \in F G^{N}$ is convex game, then the repeated fuzzy cooperative $\mathrm{w}$ is convex game with satisfies

(i) Supermodular property $\quad w(\theta \bigcup \psi)+w((\theta \cap \psi)=w(\theta)+w(\psi)$

(ii) Coordinate-wise function Let $w\left(S^{-i} \| z\right)=f_{s^{-i}}(z)$ for each $i \in N$. The t-stage game function

$f_{s^{-i}}^{t}:[0,1]^{N} \rightarrow R$ with $f_{s^{-i}}^{t}(z)=v\left(s^{-i} t \| z\right)$ be a convex function and continue in $[0,1]$.

Proof: See reference Gao Zuo-feng (2006).

The next lemma for repeated convex fuzzy game is related with the increasing marginal contribution property for players. It states that a level increase of a player in a repeated fuzzy coalition sequence has more beneficial effect in a larger coalition than in a small coalition sequences. For $\theta=\left(S^{t}\right)_{t=0}^{m} \in H, \psi=\left(T^{t}\right)_{t=0}^{m} \in H$, we use the notation $S^{t} \leq T^{t} \quad$ iff $\quad S_{i}^{t} \leq T_{i}^{t}$ for each $i \in N$.

Lemma: Let $w \in\left(F G_{t}{ }^{N}\right)_{t=0}^{m}$ be a repeated convex fuzzy cooperative games. Let $i \in N \quad \theta, \psi \in H$ with $\theta \leq \psi$ and let $\varepsilon^{t} \in R_{+}, \quad t=0,1 \cdots m$ with $0 \leq \varepsilon^{t} \leq 1-T^{t}$ Then

$$
w\left(\theta+X_{t=0}^{m} \varepsilon^{t} e^{i}\right)-w(\theta) \leq w\left(\psi+X_{t=0}^{m} \varepsilon^{t} e^{i}\right)-w(\psi)
$$

Proof: Suppose $_{N}=\{1,2 \cdots n\}$, Define the fuzzy coalition sequence of t-stage games $P_{0}^{t}, P_{1}^{t}, \cdots P_{n}^{t}$ by $P_{0}^{t}=S^{t}$ and $P_{k}^{t}=P_{k-1}^{t}+\left(S_{k}^{t}-T_{k}^{t}\right) e^{k}$ for $k=\{1,2, \cdots n\}$. Then $P_{n}^{t}=T^{t}$. To prove (4) it is sufficient to show that for each $k=\{1,2, \cdots n\}$ the inequality $\left(I^{k}\right)$ holds

$$
v\left(P_{k}^{t}+X_{t=0}^{m} \varepsilon^{t} e^{i}\right)-v\left(P_{k}^{t}\right) \geq v\left(P_{k-1}^{t}+X_{t=0}^{m} \varepsilon^{t} e^{i}\right)-v\left(P_{k-1}^{t}\right)
$$

Note that $\left(I^{k}\right)$ follows from coordinate-wise convexity of $\mathrm{w}$ and $\left(I^{k}\right)$ for $(k \neq i)$, from the supermodularity property with $P_{k-1}^{t}+\varepsilon^{t} e^{t}$ in the role of $S^{t}$ and $P_{k}^{t}$ in the role of $T^{t}$, Then $S^{t} \cup T^{t}=P_{k}^{t}+\varepsilon^{t} e^{i}, S^{t} \cap T^{t}=P_{k-1}^{t}$,

$$
\begin{aligned}
& \left.w\left(\theta+X_{t=0}^{m} \varepsilon^{t} e^{i}\right)-w(\theta)\right)=(1-\delta) \sum_{t=0}^{m} \delta^{t}\left[v\left(S^{t}+\varepsilon^{t} e^{i}\right)-v\left(S^{t}\right)\right]=(1-\delta) \sum_{t=0}^{m} \delta^{t}\left[v\left(P_{0}^{t}+\varepsilon^{t} e^{i}\right)-v\left(P_{0}^{t}\right)\right] \\
& \leq(1-\delta) \sum_{t=0}^{m} \delta^{t}\left[v\left(P_{1}^{t}+\varepsilon^{t} e^{i}\right)-v\left(P_{1}^{t}\right)\right] \leq \cdots \leq(1-\delta) \sum_{t=0}^{m} \delta^{t}\left[v\left(P_{n}^{t}+\varepsilon^{t} e^{i}\right)-v\left(P_{n}^{t}\right)\right] \\
& =(1-\delta) \sum_{t=0}^{m} \delta^{t}\left[v\left(T^{t}+\varepsilon^{t} e^{i}\right)-v\left(T^{t}\right)\right] \\
& =w\left(\psi+X_{t=0}^{m} \varepsilon^{t} e^{i}\right)-w(\psi) .
\end{aligned}
$$

The next theory introduces a characterizing property for repeated convex fuzzy games. Similar to fuzzy game, we also call the increasing average marginal return property (IAMR-Property).

Theorem 2: Let $w \in\left(F G_{t}{ }^{N}\right)_{t=0}^{m}$ be a repeated convex fuzzy game, let $i \in N, \theta, \psi \in H$, with $\theta \leq \psi$ and let 
$S_{i}^{t}+\varepsilon_{1}^{t} \leq T_{i}^{t}+\varepsilon_{2}^{t} \leq 1$ then

$$
\frac{1}{\sum_{t=0}^{m} \varepsilon_{1}^{t}}\left[w\left(\theta+X_{t=0}^{m} \varepsilon_{1}^{t} e^{i}\right)-w(\theta)\right] \leq \frac{1}{\sum_{t=0}^{m} \varepsilon_{2}^{t}}\left[w\left(\psi+X_{t=0}^{m} \varepsilon_{2}^{t} e^{i}\right)-w(\psi)\right]
$$

Proof: From Lemma (with $S^{t}, T^{t}+\left(S_{i}^{t}-T_{i}^{t}\right) e^{i}$ and $\varepsilon_{1}^{t}$ in the roles of $S^{t}$

$T^{t}$ and $\varepsilon$ respectively) It follows that

$$
\frac{1}{\sum_{t=0}^{m} \varepsilon_{1}^{t}}\left[w \left(\psi+X_{t=0}^{m}\left(S_{i}^{t}-T_{i}^{t}+\varepsilon_{1}^{t}\right) e^{i}-w\left(\psi+X_{t=0}^{m}\left(S_{i}^{t}-T_{i}^{t}\right) e^{i}\right] \geq \frac{1}{\sum_{t=0}^{m} \varepsilon_{1}^{t}}\left[w\left(\theta+X_{t=0}^{m} \varepsilon_{1}^{t} e^{i}\right)-w(\theta)\right]\right.\right.
$$

Further, from the coordinate-wise convexity (by noting $T_{i}^{t}+\left(S_{i}^{t}-T_{i}^{t}+\varepsilon_{1}^{t}\right) \leq T_{i}^{t}+\varepsilon_{2}^{t}$

$\left.T_{i}^{t}+\left(S_{i}^{t}-T_{i}^{t}\right) \leq T_{i}^{t}\right)$ it follows that

$\frac{1}{\sum_{t=0}^{m} \varepsilon_{2}^{t}}\left[w\left(\psi+X_{t=0}^{m} \varepsilon_{2}^{t} e^{i}\right)-w(\psi)\right] \geq \frac{1}{\sum_{t=0}^{m} \varepsilon_{1}^{t}}\left[w\left(\psi+X_{t=0}^{m}\left(S_{i}^{t}-T_{i}^{t}+\varepsilon_{1}^{t}\right) e^{i}-w\left(\psi+X_{t=0}^{m}\left(S_{i}^{t}-T_{i}^{t}\right) e^{i}\right]\right.\right.$

$\geq \frac{1}{\sum_{t=0}^{m} \varepsilon_{1}^{t}}\left(w\left(\theta+X_{t=0}^{m} \varepsilon_{1}^{t} e^{i}\right)-w(\theta)\right)$,resulting in the desired inequality.

Theorem 3 : If $w \in\left(F G_{t}^{N}\right)_{t=0}^{m}$. Then the following assertions are equivalent:

(i) $\mathrm{W}$ is a convex games

(ii) w satisfies the increasing average marginal return property of repeated fuzzy cooperative games.

Proof: We know from theorem that convex game satisfies IAMR-property. On the other hand, it is clear that IAMR-property implies the coordinate-wise convexity property. Hence, we only have to prove that the IAMR-property implies the supermodularity property. So given $\theta=\left(S^{t}\right)_{t=0}^{m} \in H \quad \psi=\left(T^{t}\right)_{t=0}^{m} \in H$, we only prove the supermodularity inequality (2) holds.

Let $P^{t}=\left\{i \in N \mid T_{i}^{t}<S_{i}^{t}\right\}$. If $P^{t}=\phi$, then (2) follows from the fact that $S^{t} \cup T^{t}=T^{t}$,

$S^{t} \cap T^{t}=S^{t}$. For $P^{t} \neq \phi$, arrange the elements of $P^{t}$ in t-stage as a sequence $\sigma_{t}(1), \sigma_{t}(2), \cdots$,

$\sigma_{t}\left(P^{t}\right)$, where $P^{t}=\left|P^{t}\right|$, and put $\varepsilon_{\sigma_{t}(k)}^{t}=S_{\sigma_{t}(k)}^{t}-T_{\sigma_{t}(k)}^{t}>0$ for $k \in\left\{1,2 \cdots P^{t}\right\}$. Note that in this case $S^{t}=S^{t} \cap T^{t}+\sum_{k=1}^{P^{t}} \varepsilon_{\sigma_{t}(k)}^{t} e^{\sigma_{t}(k)}, S^{t} \cup T^{t}=T^{t}+\sum_{k=1}^{P^{t}} \varepsilon_{\sigma_{t}(k)}^{t} e^{\sigma_{t}(k)}$

Hence,

$$
\begin{aligned}
& w(\theta)-w(\theta \bigcap \psi)=\sum_{r=1}^{P^{t}}\left[w\left(\theta \bigcap \psi+\sum_{k=1}^{r} X_{t=0}^{m} \varepsilon_{\sigma_{t}(k)}^{t} e^{\sigma_{t}(k)}\right)-w\left(\theta \bigcap \psi+\sum_{k=1}^{r-1} X_{t=0}^{m} \varepsilon_{\sigma_{t}(k)}^{t} e^{\sigma_{t}(k)}\right)\right] \\
& w(\theta \bigcup \psi)-w(\psi)=\sum_{r=1}^{P^{t}}\left[w\left(\psi+\sum_{k=1}^{r} X_{t=0}^{m} \varepsilon_{\sigma_{t}(k)}^{t} e^{\sigma_{t}(k)}\right)-w\left(\psi+\sum_{k=1}^{r-1} X_{t=0}^{m} \varepsilon_{\sigma_{t}(k)}^{t} e^{\sigma_{t}(k)}\right)\right]
\end{aligned}
$$

From these equalities the supermodularity inequality (2) follows because the IAMR-property implies for each $r \in\left\{1,2 \cdots P^{t}\right\}$ :

$$
w\left(\theta \bigcap \psi+\sum_{k=1}^{r} X_{t=0}^{m} \varepsilon_{\sigma_{t}(k)}^{t} e^{\sigma_{t}(k)}\right)-w\left(\theta \bigcap \psi+\sum_{k=1}^{r-1} X_{t=0}^{m} \varepsilon_{\sigma_{t}(k)}^{t} e^{\sigma_{t}(k)}\right) \leq w\left(\psi+\sum_{k=1}^{r} X_{t=0}^{m} \varepsilon_{\sigma_{t}(k)}^{t} e^{\sigma_{t}(k)}\right)-w\left(\psi+\sum_{k=1}^{r-1} X_{t=0}^{m} \varepsilon_{\sigma_{t}(k)}^{t} e^{\sigma_{t}(k)}\right) .
$$

\section{References}

Gao, Zuo feng. et al. (2006). The cores and the stable for repeated fuzzy cooperative game. Operations and research and management science. 2006, 15(4); 68-72.

J. P. Aubin. (1981). Cooperative fuzzy games. Mathematics of Operations Research.1981, (6): 1-13.

Jorge Oviedo. (2000). The core of a repeated n-person cooperative game, European Journal of Operational Research $127,2000,519-524$.

R.Branzei, D. Dimitrov, S. Tijs. (2003). Convex fuzzy games and participation monotonic allocation. schemes Center DP2002-13. Tilburg University. The Netherlands. 2002, Fuzzy Sets and Systems 139(2003) 267-281.

Xie, Z. (2004). Game Theory. ChangSha: National University of Defense Technology Press. 